\title{
The Function of Rac Small GTPase and Associated Proteins in Rice Innate Immunity
}

\author{
Yoji Kawano $\cdot$ Letian Chen $\cdot$ Ko Shimamoto
}

Received: 8 June 2010 /Accepted: 29 July 2010 /Published online: 15 August 2010

(C) Springer Science+Business Media, LLC 2010

\begin{abstract}
Two types of innate immune receptors, pattern recognition receptors, and resistance proteins, play crucial roles in plant innate immunity; however, the molecules activated by the receptors and how immune responses are transmitted are not well understood. Evidence has been accumulating for a decade that Rac, a small guanosine triphosphatase (GTPase; also known as Rop) belonging to the Rho-type small GTPase family, is a key regulator of innate immunity in rice, barley, and other species. Like other small GTPases, Rac GTPases function as molecular switches by cycling between GDP-bound inactive and GTP-bound active forms in cells. Rac GTPase acts as a key signaling switch downstream of the two types of immune receptors and triggers innate immunity. This review outlines the role of the Rac family small GTPase and its associated proteins in rice innate immunity.
\end{abstract}

Keywords Plant immunity $\cdot \mathrm{G}$ protein $\cdot$ Rice

\section{Introduction}

Plants have evolved a two-branched system of innate immunity to prevent the invasion of pathogens. Pattern recognition receptors (PRRs) are the first layer of defense against pathogen infection at the cell surface (Jones and Dangl 2006). Pathogen-specific molecules recognized by PRRs are called pathogen-associated molecular patterns (PAMPs) (Chisholm et al. 2006; Zipfel 2008). In plants,

Y. Kawano $\cdot$ L. Chen $\cdot$ K. Shimamoto $(\bowtie)$

Laboratory of Plant Molecular Genetics, Nara Institute of Science and Technology,

8916-5 Takayama, Ikoma,

Nara 630-0101, Japan

e-mail: simamoto@bs.naist.jp host perception of PAMPs activates rapid defense responses such as calcium influx, production of reactive oxygen species (ROS), induction of defense-related genes, and accumulation of antimicrobial compounds. These immunoresponses are called PAMP-triggered immunity (PTI) (Fig. 1). Most PRRs characterized to date are receptor-like kinases (RLKs) or receptor-like proteins (RLPs). RLKs possess an extracellular domain, a transmembrane domain, and a kinase domain, whereas RLPs lack the intracellular kinase domain. Protein structural analyses indicate that RLKs perceive signals through their extracellular domain and transmit signals via their intracellular kinase domain. Arabidopsis and rice encode more than 600 and 1,100 RLK/Ps, respectively (Shiu et al. 2004) that are involved in numerous cellular signaling and developmental events.

If a pathogen evades the first line of defense, it must overcome a second line of defense to become pathogenic. This defense system is termed effector-triggered immunity (ETI) (Chisholm et al. 2006; Jones and Dangl 2006) (Fig. 1). Although relying solely on germline-encoded molecules, ETI provides a remarkable level of disease resistance that rivals both the specificity and the range of mammalian adaptive immunity. ETI is triggered by disease resistance $(\mathrm{R})$ proteins that act as intracellular receptors for the direct or indirect recognition of specific pathogen effectors (also called avirulence (Avr) proteins). R proteinmediated disease resistance results in strong host responses, often culminating in a hypersensitive response (HR) and the production of ROS (Heath 2000). The bi-phasic production of apoplastic ROS, the so-called oxidative burst, is a hallmark of successful recognition of plant pathogens and a key component of the plant defense response during an incompatible interaction.

It is likely that there is the interaction between PTI and ETI (Shang et al. 2006). The Pseudomonas syringae 




Fig. 1 Model of plant innate immunity. PRRs are the first layer of defense against pathogen infection at the cell surface. PRRs recognize PAMPs and then trigger PTI. R proteins act as intracellular receptors for the direct or indirect recognition of specific pathogen effectors (also called avirulence (Avr) proteins) and induce ETI.

effector AvrB suppresses PTI through RAR1, a cochaperone of HSP90 required for ETI. AvrB expressed in plants lacking the cognate resistance gene RPM1 suppresses cell wall defense induced by the flagellar peptide flg22, a well-known PAMP, and promotes the growth of nonpathogenic bacteria in a RAR1-dependent manner. rarl mutants display enhanced cell wall defense in response to flg22, indicating that RAR1 negatively regulates PTI. It is well established that the two types of receptors (PRRs and $\mathrm{R}$ proteins) play crucial roles in plant innate immunity; however, the molecules activated by the receptors and how immune responses are transmitted remain largely unknown. Evidence has been accumulating that the Rac family small guanosine triphosphatase (GTPase) in rice is the key regulator of plant innate immunity.

\section{Rac family small GTPase in rice}

The Rho family GTPases belong to the Ras superfamily of small GTPases. The Rho family in higher eukaryotes is divided into three major subfamilies; the Rho, Rac, and Cdc42 proteins. In plants, however, the Rho family is restricted to one large family of Rac-like proteins (the Rac family). The other group named this large family Rop (RHO-related proteins from plants), therefore, the Rac family is also known as the Rop family ( $\mathrm{Li}$ et al. 1998). Like other small GTPases, members of the Rac family work as molecular switches by cycling between guanosine diphosphate (GDP)-bound inactive and guanosine triphosphate (GTP)-bound active forms in cells. Rac family members participate in various cellular events through their specific downstream effectors. In rice, there are seven genes in the Rac family, OsRac1-OsRac7 (Miki et al. 2005), whereas 11 members are found in Arabidopsis (Winge et al. 2000). The Rac family is one of the most important regulators of signal transduction in plants, participating in pathways that influence growth and development, and the adaptation of plants to various environmental situations (Berken 2006). The Rac family proteins contain five conserved regions required for GTP/GDP binding and GTP hydrolysis. All seven members of the rice Rac family (OsRac1-7) are expressed in seedlings, leaf sheaths, stems, and roots, but expression of OsRac2, 6, and 7 is rather low in leaf blades (Chen et al. 2010b). The expression level of OsRac7 is also low in panicles, immature seeds, and cultured cells. The tissue specificity of Rac/Rop expression suggests distinct roles for different Rac/Rop small GTPases in the various signaling pathways in rice.

A polybasic region and a post-translational modification site at the $\mathrm{C}$ terminus are important for membrane association and signaling functions of small GTPases. Rac/Rops can be divided into two types based on their Cterminal motifs. Type I Rac/Rops possess a conserved CaaL (a, aliphatic amino acid) motif, whereas Type II proteins lack this motif but retain a cysteine-containing element for membrane anchoring (Winge et al. 2000). Amino acid analysis of the polybasic region in seven Rac/Rop members in rice revealed that three members (OsRac5-7) are type I $\mathrm{Rac} / \mathrm{Rop}$ proteins that possess a conserved CaaL motif at the $\mathrm{C}$ terminus (Chen et al. 2010b). The remaining four (OsRac1-4) are type II Rac/Rop proteins that carry a truncated but functional post-translational modification motif. Generally, Rac/Rop proteins are predominantly localized at the plasma membrane, but some signals are observed in the nucleus and the cytoplasm (Chen et al. 2010b). Fewer type I Rac/Rops (OsRac5-7) are localized in the nucleus and the cytoplasm than type II proteins (OsRac1-4). Most constitutively active (CA) forms of Rac/Rops show a higher frequency of plasma membrane localization patterns than their dominant negative (DN) forms. OsRac3 and OsRac4 show the highest percentage of plasma membrane localization signals among rice Rac/Rop GTPases (Chen et al. 2010b).

We have previously reported that OsRac1 positively regulates disease resistance (Chen et al. 2010b; Kawano et al. 2010; Ono et al. 2001). To elucidate roles of all seven Rac family proteins in rice innate immunity, we have recently made the RNAi-mediated knockdown of the OsRac family genes and performed the infection assay (Chen et al. 2010b). OsRac4 and OsRac5 appears to be negative regulator of blast resistance because the OsRac4 and OsRac5 RNAi plants decreased the lesion lengthinduced by a virulent rice blast pathogen. These positive and negative regulators may function antagonistically in disease resistance pathway to fine tune the defense responses. There are no detectable effects in OsRac7, 
OsRac6, and OsRac3 RNAi plants. Since OsRac3 and OsRac4 are predominantly localized in the plasma membrane, there is no obvious correlation between defense function and its subcellular localization pattern. OsRac6 plays a modest role in defense based on infection studies with OsRac6 RNAi plants; however, based on an overexpression study, OsRac6, also known as $O s R a c B$, was proposed to be a negative regulator in defense (Jung et al. 2006). The existence of positive and negative roles for Rac/ Rop GTPases in rice innate immunity also suggests the complexity of Rac/Rop functions in disease resistance.

\section{Regulators of Rac GTPase}

The Rac family works as a molecular switch by cycling between GDP-bound inactive (GDP-Rac) and GTP-bound active (GTP-Rac) forms (Fig. 2). The ratio of these two forms of Rac depends on the activity of regulating factors. GTPase-activating protein acts as a negative regulator by accelerating the intrinsic GTPase activity of Rac and reconverting it to the inactive GDP-Rac. Guanine nucleotide dissociation inhibitor inhibits the exchange of GDP for GTP. Guanine nucleotide exchange factor (GEF) facilitates the release of GDP from Rac, thereby promoting the binding of GTP. GTP-Rac interacts with effectors and then triggers various cellular responses (Fig. 2).

More than 30 RacGEFs have been described in animals; most of them share conserved Dbl homology (DH homology) and pleckstrin homology (PH) domains (Bos et al. 2007); however, no Dbl homology-associated pleckstrin homology (DH-PH) RacGEFs have been found in plants. Instead, plants possess a unique family of RacGEFs whose members have clearly been demonstrated to specifically activate Rac/ROP small G proteins in vitro (Berken et

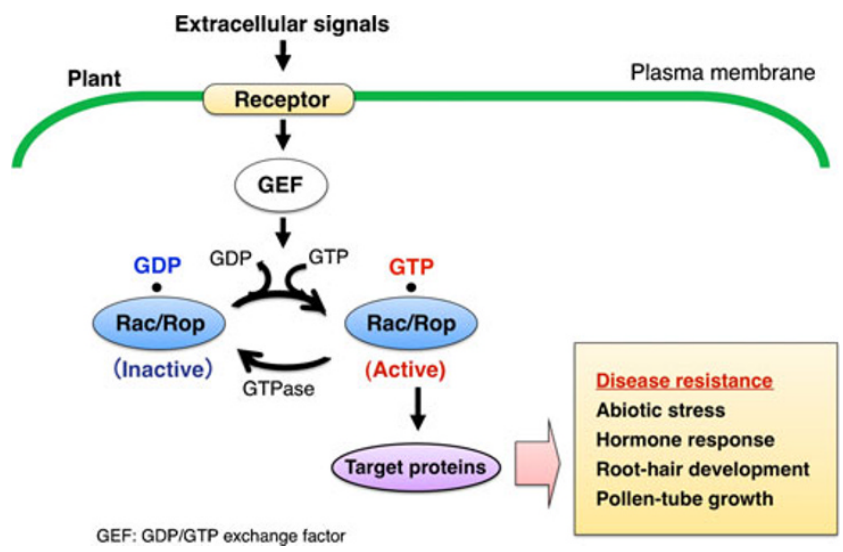

Fig. 2 Function of Rac/Rop small GTPases in plants. Small GTPases in the Rac family work as molecular switches by cycling between GDP-bound inactive and GTP-bound active forms in cells. The active GTP-bound form of Rac proteins bind to specific downstream effectors and thereby participate in various cellular events. al. 2005). The primary structure of plant RacGEFs is characterized by a highly conserved catalytic domain designated a plant-specific ROP nucleotide exchanger (PRONE). PRONE was found to stimulate nucleotide dissociation from ROP with catalytic properties comparable to DH-PH GEFs. Based on the three-dimensional structure of PRONE GEF, catalysis follows a push and pull mechanism affecting the switch regions of small GTPase (Berken and Wittinghofer 2008; Thomas et al. 2007).

\section{Upstream signals of Rac GTPase in rice innate immunity}

\section{Resistance protein}

$\mathrm{R}$ proteins act as intracellular receptors for the recognition of specific pathogen effectors (Fig. 1). Most R proteins belong to the nucleotide-binding domain (NB) and leucinerich repeat (LRR)-containing gene family (NLR, also called NB-LRR), whose members display a tripartite domain architecture consisting of an $\mathrm{N}$-terminal variable region, a central NB domain and C-terminal LRRs. However, there are structural variations of $\mathrm{R}$ protein. $\mathrm{Cf}-9$ which is a $\mathrm{R}$ protein to Cladosporium fulvum resembles the membrane bound receptor domain of RLKs but lacks the protein kinase domain. Conversely, the tomato Pto gene for resistance to $P$. syringae $p v$. tomato encodes a protein kinase resembling the membrane bound kinase domain of RLKs but lacking the extracellular domain.

The NB domain is part of a larger domain called NBARC (ARC: APAF-1, certain R gene products and CED-4). Several lines of evidence suggest that the N-terminal variable region of most NLR family R proteins participates in indirect pathogen recognition. It is likely that some functions of these N-terminal variable regions differ between plants and animals (DeYoung and Innes 2006; Lukasik and Takken 2009). A strong HR immune response can be induced by the coiled-coil (CC) domain and NBARC fragments (CC-NB-ARC) of the NLR family $\mathrm{R}$ proteins RPS2 and RPS5 (Ade et al. 2007; Tao et al. 2000), whereas the Toll/interleukin-1 receptor (TIR) domain and the NB-ARC fragments (TIR-NB-ARC) of NLR family R proteins RPS4 and RPP1A are sufficient to induce the HR (Michael Weaver et al. 2006; Zhang et al. 2004). Moreover, expression of the NB fragment of potato NLR family R protein Rx induces the HR (Rairdan et al. 2008). These and other results indicate that the NB-ARC domain of NLR family R proteins serves as a platform leading to downstream signal transduction events (Lukasik and Takken 2009; Takken et al. 2006). In plants, it remains largely unknown which signal transducers transmit the signals from NLR family $\mathrm{R}$ proteins to trigger immune 
responses such as the HR and ROS production. To gain a deeper understanding of how NLR family R proteins regulate plant defenses, it is essential to identify the partners that interact with the NB-ARC domain (Takken and Tameling 2009).

We recently found that OsRac1 is required for $P i-a$ mediated defense responses (Chen et al. 2010b). Pi-a is one of the resistance genes to rice blast fungus. Moreover, another research group has overexpressed DN-OsRac1 in transgenic tobacco carrying the $N$ resistance gene and tested its effects (Moeder et al. 2005). DN-OsRac1 suppresses the synchronous HR and ROS production triggered by $N$ as well as Pto resistance genes. In addition, we recently found that OsRac1 interacts directly with the NB-ARC domain of Pit, an NLR family disease resistance protein that confers resistance to rice blast fungus, at the plasma membrane. OsRac1 contributes to Pit-mediated ROS production as well as the HR and is required for Pitmediated disease resistance in rice. Furthermore, in vivo Förster resonance energy transfer (FRET) experiments indicated that the active form of Pit induces the activation of OsRac1 at the plasma membrane. Thus, OsRac1 is activated by Pit during pathogen attack, and this activation plays a critical role in Pit-mediated immunity in rice (Fig. 3). We do not yet know whether Pit directly activates OsRac1. GEFs are believed to be the most important regulatory proteins in the activation of small GTPases (Bos et al. 2007). Pit lacks a GEF domain, but its CC-NB region shows detectable sequence similarity to the $\mathrm{DH}$ domains of DH-PH family GEFs. Because it is difficult to purify an intact recombinant Pit protein, we have been unable to assess the GEF activity of Pit in vitro.



Fig. 3 Activation of OsRac1 by $\mathrm{R}$ protein plays a critical role in rice innate immunity. OsRac1 at the plasma membrane interacts directly with Pit, an NLR protein that confers resistance to rice blast fungus. OsRac1 contributes to Pit-mediated ROS production as well as the HR and is required for Pit-mediated disease resistance in rice. Furthermore, the active form of Pit induces the activation of OsRac1 at the plasma membrane. Thus, OsRac1 is activated by Pit during pathogen attack and plays a critical role in Pit-mediated immunity in rice.
Alternatively, Pit may function as a cofactor or an activator of a GEF for OsRac1; in this context, we recently identified a PRONE-type Rac GEF by yeast two-hybrid screening and found that it induces OsRac1 activation in vitro and in vivo (A. Akamatsu et al., unpublished data). Whether Pit participates in the activation of this OsRac1 GEF will be the subject of future studies.

\section{Pattern recognition receptor}

RLKs function in plant-microbe interactions and defense responses. FLS2 and EFR, for example, are receptors for bacterial flagellin and elongation factor $\mathrm{Tu}$, respectively (Gomez-Gomez and Boller 2000; Zipfel et al. 2006) (Fig. 1), whereas CEBiP and LysM-type CERK1 are receptors for fungal chitin (Miya et al. 2007). Recently, BRI-associated kinase (BAK1) was found to be important for innate immunity as well as cell death (Chinchilla et al. 2007), suggesting that different receptor-like kinases (RLKs) or receptor-like proteins (RLPs)-mediated signaling pathways share common components. We previously reported that the overexpression of DN-OsRac1 compromises elicitor-induced ROS production, indicating that OsRac1 acts as a downstream molecule of the RLK pathway (Ono et al. 2001). However, it has been largely unknown the signaling pathway from RLK to OsRac1 at present.

Studies in other areas provide clues for speculation about how RLK activates Rac family proteins in plant innate immunity. The Rac family has been implicated in signaling downstream of RLK CLAVATA1, part of a protein complex regulating the balance between cell differentiation and cell division in aerial meristems (Trotochaud et al. 1999). An unidentified Rop GTPase is immunologically detected in the $450 \mathrm{kDa}$ active CLAVATA1 complex. Interestingly, McCormick's group found that PRONE-type AtRopGEF interacts with pollen-specific RLKs, LePRK1 and LePRK2, implying that RopGEF activity may be regulated by RLKs (Kaothien et al. 2005). They characterized an Arabidopsis homolog of LePRK2, AtPRK2a and verified the physical interaction between AtPRK2a and the Arabidopsis PRONE-type AtRopGEF12. PRONE is flanked by variable $\mathrm{N}$ - and C-termini of RopGEFs that may be important for regulation of the proteins ( $\mathrm{Gu}$ et al. 2006). An autoinhibitory mechanism in the $\mathrm{N}$ - and $\mathrm{C}$-terminal regions was proposed for regulating Arabidopsis RopGEF1, and an intramolecular interaction between the $\mathrm{C}$ terminus and the catalytic domain may block GEF activity. It is likely that the phosphorylation of the $\mathrm{C}$ terminus of AtRopGEF12 is important for its GEF activity. A phospho-mimicking mutation at an invariant serine within the $\mathrm{C}$ terminus of AtRopGEF12 results in loss of the C-terminal inhibition. 
Taken together, these findings suggest that RopGEFphosphorylated by RLKs plays important roles in Rac family-dependent PTI (Fig. 4).

\section{Heterotrimeric $\mathrm{G}$ proteins}

Heterotrimeric G-proteins, a major group of signaling molecules involved in a variety of cellular activities in mammals, are mainly responsible for various cellular responses to external signals. In plants, a number of pharmacological studies suggested that heterotrimeric Gproteins are involved in a variety of signaling activities, including light reception, hormone signaling, and regulation of ion channels (Perfus-Barbeoch et al. 2004). Analysis of mutations in a gene encoding the $\mathrm{G} \alpha$ subunit of rice termed dwarfl ( $d 1$ ) showed that $\mathrm{G} \alpha$ is involved in stem elongation and the determination of seed shape in rice and influences gibberellin signal transduction (Fujisawa et al. 2001). Therefore, the importance and diverse functions of heterotrimeric G-proteins in plant signal transduction are being elucidated. Many studies using inhibitors and agonists of heterotrimeric G-proteins in several plant species have suggested that G-proteins are involved in defense signaling (Beffa et al. 1995; Legendre et al. 1992). Particularly, changes in cytosolic $\mathrm{Ca}^{2+}$ concentrations, often observed in elicitor-treated plant cells, are assumed to be regulated by heterotrimeric G-proteins (Aharon et al. 1998). We used rice $d 1$ mutants lacking a single-copy of the $\mathrm{G} \alpha$ gene and addressed G-proteins' role in disease resistance (Suharsono et al. 2002). $d 1$ mutants exhibited a highly reduced HR to infection by an avirulent race of rice blast and enhanced hyphal extension, indicating that $\mathrm{G} \alpha$ is involved in R-gene-



Fig. 4 OsRac1 and its associated proteins regulate rice innate immunity. The defensome is defined as a functional network of rice innate immunity consisting of four different components including immune receptors (yellow), (co)-chaperones (green), the immune switch OsRac1 (blue), and downstream effectors of OsRac1 (purple). Defensome assembles several signaling proteins together so that they may trigger immune responses quickly and efficiently. mediated disease resistance in rice, at least in rice-blast interactions. Activation of Pathogenesis-related gene $P B Z 1$ expression in the leaves of the mutants infected with rice blast is delayed for $24 \mathrm{~h}$ relative to the wild type. ROS production and $P R$ gene expression induced by sphingolipid elicitors are strongly suppressed in $d 1$ cell cultures. Expression of CA-OsRac1 in $d 1$ mutants restores sphingolipid elicitors-dependent defense signaling and resistance to rice blast, suggesting that OsRac1 is located downstream of $\mathrm{G} \alpha . G \alpha$ mRNA is induced by an avirulent race of rice blast and sphingolipid elicitors application on the leaf. Based on these results, we propose a model for rice defense signaling in which the heterotrimeric G-protein functions upstream of OsRac1 in the early steps of signaling. Another research group showed that $\mathrm{G} \alpha$ is involved in the induction of $P B Z 1$ by the plant activator Probenazol (Iwata et al. 2003). Probenazol has been widely used as a rice blast-controlling chemical during rice cultivation and is considered a plant activator because it shows no anti-fungal activity against rice blast fungus but activates the disease-defense system of the host plant. We previously found that expression of CAOsRac1 induces the expression of PBZ1. It is possible that OsRac1 is activated by Probenazol downstream of $\mathrm{G} \alpha$.

\section{Target proteins of Rac GTPase in rice innate immunity}

Nicotinamide adenine dinucleotide phosphate oxidase

In plant cells, ROS can directly cause strengthening of host cell walls via cross-linking of glycoproteins, or lipid peroxidation and membrane damage; however, it is also evident that ROS are important signals mediating defense gene activation (Torres and Dangl 2005). Additional regulatory functions for ROS in defense occur in conjunction with other plant signaling molecules, particularly salicylic acid and nitric oxide. Although numerous nicotinamide adenine dinucleotide phosphate (NADPH) oxidases (respiratory burst oxidase homolog (Rboh)) have been isolated in plants, all rboh genes identified to date possess a conserved $\mathrm{N}$-terminal extension that contains two $\mathrm{Ca}^{2+}$ binding EF-hand motifs. Mechanisms regulating enzymatic activity were largely unknown.

In 1999, we found that OsRac1 is a regulator of ROS production and cell death in rice (Kawasaki et al. 1999). CA-OsRac1 enhances PAMPs-induced ROS production and resistance to pathogens in rice (Ono et al. 2001). The interaction between OsRac1 and the $\mathrm{N}$-terminal extension is ubiquitous, and a substantial part of the N-terminal region of Rboh, including the two EF-hand motifs, is required for the interaction (Wong et al. 2007). In vivo FRET analysis also suggests that cytosolic $\mathrm{Ca}^{2+}$ concentration may regulate $\mathrm{Rac}-\mathrm{Rboh}$ interaction in a dynamic manner. 
Furthermore, the direct Rac-Rboh interaction activates NADPH oxidase activity in plants. Structure-based analysis further supports the direct interaction between OsRac1 and RbohB (Oda et al. 2010). The OsRacl binding interface of RbohB is located in the flanking region of the coiled-coil region at $\mathrm{N}$ terminus. This binding region is not similar to those previously identified as Rac binding motifs. Thus, OsRac1 interacts with OsRbohB in a manner distinct from known interactions between Rac and its target proteins. Collectively, these results suggest that cytosolic $\mathrm{Ca}^{2+}$ concentration may modulate NADPH oxidase activity by regulating the direct interaction between Rac GTPase and Rboh.

NADPH oxidases appear to be required for the oxidative burst after pathogen recognition by $\mathrm{R}$ proteins (Torres and Dangl 2005). DN-OsRacl suppresses both $\mathrm{R}$ protein Pitand N-induced ROS production (Kawano et al. 2010; Moeder et al. 2005), suggesting that OsRac1 contributes to the oxidative burst after pathogen recognition. Experiments with the FRET sensor construct Raichu-OsRac1 revealed that Pit interacts with and activates OsRac1 at the plasma membrane, where NADPH oxidases are located. Therefore, it seems that OsRac1 acts as a signal transducer from Pit to NADPH oxidase at the plasma membrane during the oxidative burst. The expression of a ROS scavenging gene Metallothionein $2 b$ was synergistically down-regulated by OsRac1 and rice blast-derived elicitors (Wong et al. 2004). Thus, OsRac1 might play a dual role as an inducer of ROS production and a suppressor of ROS scavenging.

The chaperone complex (HSP90, Hop/Sti1, RAR1, and SGT1)

$\mathrm{RLK} / \mathrm{Ps}$ are located in the plasma membrane and are assumed to move to endosomes through endocytosis; however, the modes of maturation, trafficking and plasma membrane localization of RLK/Ps are largely unknown. We recently found that the rice chitin receptor OsCERK1 interacts with HSP90 and its co-chaperone Hop/Sti1 in the endoplasmic reticulum (ER) (Chen et al. 2010a) (Fig. 5). The knockdown of Hop/Stil compromises chitin-triggered pathogenesis-related gene expression and virulent rice blast fungus, suggesting that Hop/Stil is required for chitin-triggered immunity and resistance to rice blast fungus. Hop/Sti1 and HSP90 regulate efficient transport of OsCERK1 from the ER to the plasma membrane via a pathway dependent on Sar1, a small GTPase that regulates ER-to-Golgi trafficking. These results suggest that the Hop/Sti1-HSP90 chaperone complex plays an important and likely conserved role in the maturation and transport of PRRs and may function to link PRRs and Rac/Rop GTPases.

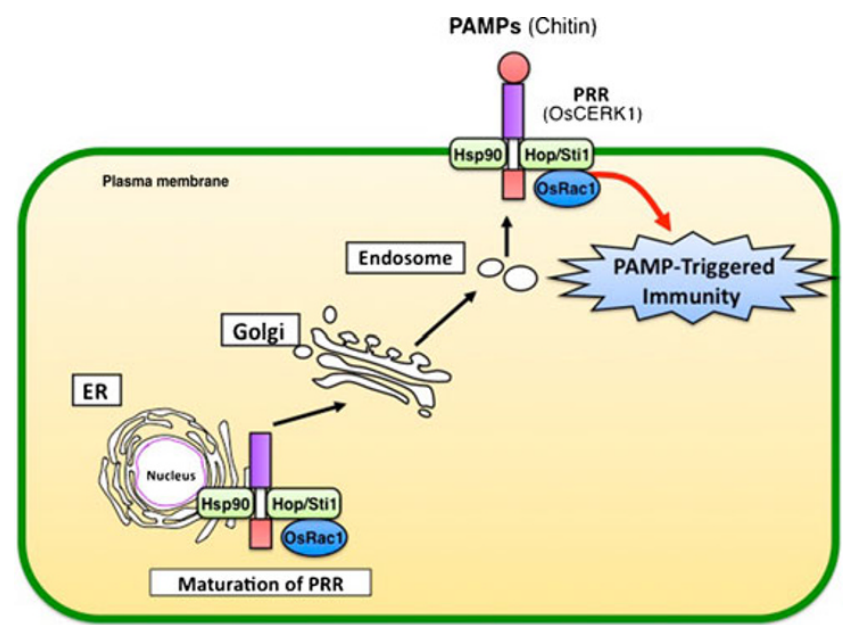

Fig. 5 The Hop/Sti1-HSP90 chaperone complex facilitates the maturation and transport of PRRs. The rice chitin receptor OsCERK1 interacts with HSP90 and its chaperone Hop/Stil in the ER. Hop/Sti1 and HSP90 are required for efficient transport of OsCERK1 from the ER to the plasma membrane via ER-to-Golgi trafficking. Further, Hop/Sti1 and HSP90 interact with OsRac1 and OsCERK1. The Hop/ Sti1-HSP90 chaperone complex plays an important and conserved role in the maturation and transport of PRRs.

In animals, Hop/Stil is best known as one of the cochaperones for the cytoplasmic HSP90 chaperone that participates in a complex that regulates steroid hormone receptor biogenesis and maturation. Cytoplasmic HSP90 and two co-chaperone-like molecules, RAR1 and SGT1, form a ternary complex and play a critical role in innate immune responses triggered by $\mathrm{R}$ proteins in Arabidopsis and tobacco (Shirasu 2009). We recently demonstrated that RAR1, HSP90, and HSP70 are present in the OsRac1 complex, but none of them appear to interact directly with OsRac1 (Thao et al. 2007). The OsRac1-interacting scaffold protein Receptor for activated C-kinase 1 (RACK1)A directly interacts with SGT1 and RAR1, but not with HSP90 (Nakashima et al. 2008). The interaction of these three (co)-chaperones seems to contribute mainly to basal resistance in rice (Thao et al. 2007; Wang et al. 2008). Although the involvement of the HSP90 chaperone complex and other co-chaperone-like proteins in plant innate immunity has been well established, the molecular mechanisms of their functions are not yet understood (Hubert et al. 2009; Shirasu 2009). We previously showed that HSP90 inhibitor geldanamycin treatment suppresses PAMPtriggered immune responses in rice cells and disrupts OsRac1-HSP90 complex formation (Thao et al. 2007). It is possible that the OsRac1-HSP90 complex is a component of a larger plasma membrane protein complex that contains RLK, Hop/Stil, and the plasma membrane-anchored OsRac1. Since Hop/Stila-RNAi and geldanamycin decreased the efficiency of the plasma membrane targeting of OsCERK1, and thereby impaired chitin-triggered de- 
fense gene expression, it seems possible that the HSP90 chaperone complex, including Hop/Stila, has a dual function in rice innate immunity; one function is related to efficient export from the ER and plasma membrane localization of PRRs, and the other to signaling in the defensome at the plasma membrane. The precise mechanisms by which these proteins regulate maturation, ER export and trafficking of OsCERK1 remain to be elucidated. Recent studies have indicated that key components of the ER quality control system are involved in innate immune responses in plants ( $\mathrm{Li}$ et al. 2009; Nekrasov et al. 2009; Saijo et al. 2009). Our results suggest that Hop/ Sti1 and HSP90 regulate OsCERK1 maturation by assembling a complex (or complexes) with OsRac1 in the ER and subsequently transporting OsCERK1 from the ER to the plasma membrane. How the ER quality control system cooperates with the HSP90-Hop/Stil chaperone machinery to regulate the maturation, ER export, and trafficking of OsCERK1 will be an interesting topic for the future.

\section{Receptor for activated C-kinase 1}

RACK1 interacts with many signaling proteins in animals and based on its structure is considered to be a scaffolding protein in a number of signaling pathways (McCahill et al. 2002). Thus, it is evident that RACK 1 plays multiple roles in the cellular activities of eukaryotes. We previously showed that rice RACK1 plays a key role in the production of ROS and disease resistance and binds RAR1 and SGT1 (Shirasu 2009). We used affinity column chromatography to identify rice RACK1 as an interactor with OsRac1 (Nakashima et al. 2008) (Fig. 4). RACK1 functions in various mammalian signaling pathways and is involved in hormone signaling and development in plants (Chen et al. 2006). Rice contains two RACK1 genes, RACK1A and RACK1B, and the RACK1A protein interacts with the GTP form of OsRac1 (Nakashima et al. 2008). OsRac1 positively regulates RACK1A at both the transcriptional and posttranscriptional levels. RACK1A transcription was also induced by a fungal elicitor and by abscisic acid, jasmonate, and auxin. Analysis of transgenic rice plants and cell cultures indicates that RACK1A plays a role in the production of ROS and in resistance against rice blast infection. Overexpression of RACK1A enhances ROS production in rice seedlings. RACK1A was shown to interact with the $\mathrm{N}$ terminus of NADPH oxidase, RAR1, and SGT1, key regulators of plant disease resistance. These results suggest that RACK1A functions in rice innate immunity by interacting with multiple proteins in the OsRac1 immune complex. Based on these results obtained in our study, two functions of RACK1 in rice innate immunity can be envisaged. One possible function is that RACK1 is a component of the OsRac1 complex consisting of OsRac1, RAR1, SGT1, HSP90, and HSP70 and functions as a scaffolding protein for the immune complex. We previously postulated that all of these proteins could form a protein complex (Thao et al. 2007). The abundance of each of the (co)-chaperones (RAR1, HSP90, and HSP70) present in the immune complex may need to be finely regulated to ensure a rapid and stable response to pathogen attack. Another hypothesis is that RACK1A is a component of the NADPH oxidase complex together with OsRac1 and regulates ROS production at an early stage of immune responses since RACK1A interacts with the N-terminal region of NADPH oxidase (Rboh). How these functions of RACK1A are regulated or how its interactions with other proteins are temporally and spatially regulated after pathogen infection remain to be studied in the future.

We recently found that OsRac1 and RACK1A shifts to the detergent-resistant membranes (DRMs) fraction after chitin elicitor treatment (Fujiwara et al. 2009). DRMs are regions of the plasma membrane that are insoluble after Triton X-100 treatment under cold conditions and are thought to be involved in numerous signaling processes in animal, yeast, and plant cells. After animal cells are stimulated with bacterial endotoxin and lipopolysaccharide, signaling components such as receptors, G-proteins, heat shock proteins and protein kinases move to and are concentrated in DRMs (Triantafilou et al. 2002; Yuyama et al. 2007). Therefore, DRMs may have a role in providing a platform for the initial events of the immune response in plants as well as in mammals.

\section{Cinnamoyl-CoA reductase1}

Lignin, a major component of secondary cell walls, is a heterogeneous tridimensional phenolic polymer resulting from the oxidative polymerization of monolignols (Boerjan et al. 2003). During defense responses, lignin and ligninlike phenolic compounds accumulate throughout the HR region. Deposition of lignin during defense responses is considered to function as a physical barrier against pathogen infection (Moerschbacher et al. 1990). Cinnamoyl-CoA esters, the precursors of monolignol biosynthesis, are generated by the general phenylpropanoid pathway and then converted into monolignols by two enzymes, cinnamoyl-CoA reductase 1 (CCR1) and cinnamyl alcohol dehydrogenase. The monolignols catalyzed by CCR and cinnamyl alcohol dehydrogenase are transferred to the cell wall and polymerized by peroxidase $\mathrm{H}_{2} \mathrm{O}_{2}$ induced as one of the defense responses may stimulate polymerization of monolignols in the infected regions. It is also possible that monolignols have antimicrobial activity, as has been previously reported (Keen and Littlefield 1979).

Rice CCR1 (OsCCR1), an enzyme involved in lignin biosynthesis, is a target protein of OsRac1 (Kawasaki et al. 
2006) (Fig. 4). Lignin is an important factor in plant defense responses because it presents an undegradable mechanical barrier to most pathogens. Expression of OsCCR1 is induced by a sphingolipid elicitor, suggesting that OsCCR1 participates in defense signaling. OsRac1 is shown to bind OsCCR1 in a GTP-dependent manner. Moreover, the interaction of OsCCR1 with OsRac1 leads to the enzymatic activation of OsCCR1 in vitro. Transgenic cell cultures expressing constitutively active OsRac1 accumulates lignin through enhanced CCR activity and increased ROS production. Thus, it is likely that OsRac1 controls lignin synthesis through regulation of both NADPH oxidase and OsCCR1 activities during defense responses.

Mitogen-activated protein kinase 6

Several MAPKKs and MAPKKKs upstream of wellcharacterized MAPKs have been identified, suggesting that MAPK cascades also operate in plant defense signaling responses. Among these, the constitutively active MAPKK NtMEK2 activates NtSIPK and NtWIPK, followed by induced HR-like cell death and defense gene expression (Yang et al. 2001). A complete MAPK cascade (involving MEKK1, MKK4/MKK5, and MPK3/MPK6) has been proposed in Arabidopsis (Asai et al. 2002). Oryza sativa mitogen-activated protein kinase 6 (OsMAPK6) protein levels are strongly reduced in OsRac1-silenced cells and in the $d 1$ mutant ( $\mathrm{G} \alpha$ mutant) and sphingolipid elicitorinduced OsMAPK6 activation is greatly reduced in these mutant cells (Lieberherr et al. 2005) (Fig. 4). These results suggest that the two GTP-binding proteins are required for the accumulation of OsMAPK6 protein and, possibly, for its activation as well. Furthermore, OsMAPK6 and OsRac1 proteins are in the same protein complex. Previous studies showed that $\mathrm{G} \alpha$ functions upstream of OsRac1 in the sphingolipid elicitor signaling pathway, leading to the induction of ROS production and defense gene expression (Suharsono et al. 2002). Therefore, a MAPK cascade may be similarly activated by these two G-proteins along with other pathways. The mechanism of how $\mathrm{G} \alpha$ activates OsRac1 in these signaling pathways remains to be studied. It is known that, in mammals and yeast, Ras-like GTPases are involved in upstream signaling for MAPK cascade activation (Dohlman and Thorner 2001; Ory and Morrison 2004; Ramezani-Rad 2003). These Ras-MAPK or Gprotein-MAPK cascades occur in response to various stimuli, such as hormones or environmental stresses. The signals are either transduced into the cascade components by direct protein interactions or require additional intermediate regulating factors in mammals; however, the mechanisms leading from two types of G-proteins to MAPK signaling in plants remains to be elucidated.

\section{Defensome model for rice innate immunity}

Extending our previous studies have shown the interactions between OsRac1 and a number of proteins; PRR (Chen et al. 2010a), R protein (Kawano et al. 2010), (co)-chaperone (Chen et al. 2010a; Thao et al. 2007), NADPHoxidase (Wong et al. 2007), CCR1 (Kawasaki et al. 2006), and MAPK6 (Lieberherr et al. 2005). Those proteins are involved in rice immune response. Recently, our gel filtration assay has revealed that OsRac1 forms a large protein complex containing those OsRac1 interactors (Chen et al. 2010a) (S. Hamada, and K. Shimamoto, unpublished data). Thus, we believe that these findings lend support to Defensome model. However, Defensome model is a possible model for OsRac1-dependnet immunity, therefore, we cannot neglect other possibilities.

The defensome seems to be a functional network in which each protein helps to process the signal in one or more ways as it spreads the signal's influence throughout the cell. The defensome consists of four different groups of proteins, including two types of immune receptors (PRRs and $\mathrm{R}$ proteins), chaperones, and co-chaperones (SGT1, RAR1, HSP90, HSP70, Hop/Sti1, and RACK1), the molecular switch OsRac1 and its activator RacGEF, and downstream target proteins of OsRac1 (NADPH oxidase, CCR1, and MAPK6). Defensome assembles several signaling proteins together so that they may trigger immune responses quickly and efficiently. We propose that the components of the defensome have two functions: one is to contribute to receptor stability/maturation/transport and the formation of the signaling complex and the other is to trigger signaling transduction and immune response at the plasma membrane after sensing pathogens (Chen et al. 2010a). A better understanding of the molecular roles of immune complexes containing receptors, OsRac1, and chaperones is becoming increasingly important for the study of innate immunity in plants.

Acknowledgement This research was supported by Grants-in-Aid from the Ministry of Agriculture, Forestry, and Fisheries of Japan (Genomics for Agricultural Innovation, PMI-0007) and the Japan Society for the Promotion of Science (13G0023) to K.S. and the Naito Foundation and Maekawa Houonkai Foundation to Y.K.

\section{References}

Ade J, DeYoung BJ, Golstein C, Innes RW. Indirect activation of a plant nucleotide binding site-leucine-rich repeat protein by a bacterial protease. Proc Natl Acad Sci USA. 2007;104:2531-6.

Aharon GS, Gelli A, Snedden WA, Blumwald E. Activation of a plant plasma membrane $\mathrm{Ca} 2+$ channel by TGalpha1, a heterotrimeric $\mathrm{G}$ protein alpha-subunit homologue. FEBS Lett. 1998;424:17-21.

Asai T, Tena G, Plotnikova J, Willmann MR, Chiu WL, GomezGomez L, et al. MAP kinase signalling cascade in Arabidopsis innate immunity. Nature. 2002;415:977-83. 
Beffa R, Szell M, Meuwly P, Pay A, Vogeli-Lange R, Metraux JP, et al. Cholera toxin elevates pathogen resistance and induces pathogenesis-related gene expression in tobacco. EMBO J. 1995;14:5753-61.

Berken A. ROPs in the spotlight of plant signal transduction. Cell Mol Life Sci. 2006;63:2446-59.

Berken A, Wittinghofer A. Structure and function of Rho-type molecular switches in plants. Plant Physiol Biochem. 2008;46:380-93.

Berken A, Thomas C, Wittinghofer A. A new family of RhoGEFs activates the Rop molecular switch in plants. Nature. 2005;436:1176-80.

Boerjan W, Ralph J, Baucher M. Lignin biosynthesis. Annu Rev Plant Biol. 2003;54:519-46.

Bos JL, Rehmann H, Wittinghofer A. GEFs and GAPs: critical elements in the control of small G proteins. Cell. 2007;129:86577.

Chen JG, Ullah H, Temple B, Liang J, Guo J, Alonso JM, et al. RACK1 mediates multiple hormone responsiveness and developmental processes in Arabidopsis. J Exp Bot. 2006;57:2697708 .

Chen L, Hamada S, Fujiwara M, Zhu T, Thao NP, Wong HL, et al. The Hop/Sti1-Hsp90 chaperone complex facilitates the maturation and transport of a PAMP receptor in rice innate immunity. Cell Host Microbe. 2010a;7:185-96.

Chen L, Shiotani K, Togashi T, Miki D, Aoyama M, Wong HL, et al. Analysis of the Rac/Rop small GTPase family in rice: expression, subcellular localization and role in disease resistance. Plant Cell Physiol. 2010b;51:585-95.

Chinchilla D, Zipfel C, Robatzek S, Kemmerling B, Nurnberger T, Jones JD, et al. A flagellin-induced complex of the receptor FLS2 and BAK1 initiates plant defence. Nature. 2007;448:497-500.

Chisholm ST, Coaker G, Day B, Staskawicz BJ. Host-microbe interactions: shaping the evolution of the plant immune response. Cell. 2006;124:803-14.

DeYoung BJ, Innes RW. Plant NBS-LRR proteins in pathogen sensing and host defense. Nat Immunol. 2006;7:1243-9.

Dohlman HG, Thorner JW. Regulation of G protein-initiated signal transduction in yeast: paradigms and principles. Annu Rev Biochem. 2001;70:703-54.

Fujisawa Y, Kato H, Iwasaki Y. Structure and function of heterotrimeric G proteins in plants. Plant Cell Physiol. 2001;42:789-94.

Fujiwara M, Hamada S, Hiratsuka M, Fukao Y, Kawasaki T, Shimamoto K. Proteome analysis of detergent-resistant membranes (DRMs) associated with OsRac1-mediated innate immunity in rice. Plant Cell Physiol. 2009;50:1191-200.

Gomez-Gomez L, Boller T. FLS2: an LRR receptor-like kinase involved in the perception of the bacterial elicitor flagellin in Arabidopsis. Mol Cell. 2000;5:1003-11.

$\mathrm{Gu}$ Y, Li S, Lord EM, Yang Z. Members of a novel class of Arabidopsis Rho guanine nucleotide exchange factors control Rho GTPasedependent polar growth. Plant Cell. 2006;18:366-81.

Heath MC. Hypersensitive response-related death. Plant Mol Biol. 2000;44:321-34.

Hubert DA, He Y, McNulty BC, Tornero P, Dangl JL. Specific Arabidopsis HSP90.2 alleles recapitulate RAR1 cochaperone function in plant NB-LRR disease resistance protein regulation. Proc Natl Acad Sci USA. 2009;106:9556-63.

Iwata M, Umemura K, Teraoka T, Usami H, Fujisawa Y, Iwasaki Y. Role of the $\alpha$ subunit of heterotrimeric G-protein in probenazoleinducing defense signaling in rice. J Gen Plant Pathol. 2003;69:83-6.

Jones JD, Dangl JL. The plant immune system. Nature. 2006;444:323-9.

Jung YH, Agrawal GK, Rakwal R, Kim JA, Lee MO, Choi PG, et al. Functional characterization of OsRacB GTPase - a potentially negative regulator of basal disease resistance in rice. Plant Physiol Biochem. 2006;44:68-77.

Kaothien P, Ok SH, Shuai B, Wengier D, Cotter R, Kelley D, et al. Kinase partner protein interacts with the LePRK1 and LePRK2 receptor kinases and plays a role in polarized pollen tube growth. Plant J. 2005;42:492-503.

Kawano Y, Akamatsu A, Hayashi K, Housen Y, Okuda J, Yao A, et al. Activation of a Rac GTPase by the NLR family disease resistance protein Pit plays a critical role in rice innate immunity. Cell Host Microbe. 2010;7:362-75.

Kawasaki T, Henmi K, Ono E, Hatakeyama S, Iwano M, Satoh H, et al. The small GTP-binding protein Rac is a regulator of cell death in plants. Proc Natl Acad Sci USA. 1999;96:10922-6.

Kawasaki T, Koita H, Nakatsubo T, Hasegawa K, Wakabayashi K, Takahashi H, et al. Cinnamoyl-CoA reductase, a key enzyme in lignin biosynthesis, is an effector of small GTPase Rac in defense signaling in rice. Proc Natl Acad Sci USA. 2006;103:230-5.

Keen NT, Littlefield LJ. The possible association of phytoalexins with resistance gene expression in flax to Melampsora lini. Physiol Plant Pathol. 1979;14:265-80.

Legendre L, Heinstein PF, Low PS. Evidence for participation of GTP-binding proteins in elicitation of the rapid oxidative burst in cultured soybean cells. J Biol Chem. 1992;267:20140-7.

Li H, Wu G, Ware D, Davis KR, Yang Z. Arabidopsis Rho-related GTPases: differential gene expression in pollen and polar localization in fission yeast. Plant Physiol. 1998;118:407-17.

Li J, Zhao-Hui C, Batoux M, Nekrasov V, Roux M, Chinchilla D, et al. Specific ER quality control components required for biogenesis of the plant innate immune receptor EFR. Proc Natl Acad Sci USA. 2009;106:15973-8.

Lieberherr D, Thao NP, Nakashima A, Umemura K, Kawasaki T, Shimamoto K. A sphingolipid elicitor-inducible mitogenactivated protein kinase is regulated by the small GTPase OsRac1 and heterotrimeric G-protein in rice 1. Plant Physiol. 2005; 138:1644-52.

Lukasik E, Takken FL. STANDing strong, resistance proteins instigators of plant defence. Curr Opin Plant Biol. 2009;12:427-36.

McCahill A, Warwicker J, Bolger GB, Houslay MD, Yarwood SJ. The RACK1 scaffold protein: a dynamic cog in cell response mechanisms. Mol Pharmacol. 2002;62:1261-73.

Michael Weaver L, Swiderski MR, Li Y, Jones JD. The Arabidopsis thaliana TIR-NB-LRR R-protein, RPP1A; protein localization and constitutive activation of defence by truncated alleles in tobacco and Arabidopsis. Plant J. 2006;47:829-40.

Miki D, Itoh R, Shimamoto K. RNA silencing of single and multiple members in a gene family of rice. Plant Physiol. 2005;138:190313.

Miya A, Albert P, Shinya T, Desaki Y, Ichimura K, Shirasu K, et al. CERK1, a LysM receptor kinase, is essential for chitin elicitor signaling in Arabidopsis. Proc Natl Acad Sci USA. 2007;104:196138.

Moeder W, Yoshioka K, Klessig DF. Involvement of the small GTPase Rac in the defense responses of tobacco to pathogens. Mol PlantMicrob Interact. 2005;18:116-24.

Moerschbacher BM, Noll U, Gorrichon L, Reisener HJ. Specific inhibition of lignification breaks hypersensitive resistance of wheat to stem rust. Plant Physiol. 1990;93:465-70.

Nakashima A, Chen L, Thao NP, Fujiwara M, Wong HL, Kuwano M, et al. RACK1 functions in rice innate immunity by interacting with the Rac1 immune complex. Plant Cell. 2008;20:2265-79.

Nekrasov V, Li J, Batoux M, Roux M, Chu ZH, Lacombe S, et al. Control of the pattern-recognition receptor EFR by an ER protein complex in plant immunity. EMBO J. 2009;28:3428-38.

Oda T, Hashimoto H, Kuwabara N, Akashi S, Hayashi K, Kojima C, et al. Structure of the N-terminal regulatory domain of a plant 
NADPH oxidase and its functional implications. J Biol Chem. 2010;285:1435-45.

Ono E, Wong HL, Kawasaki T, Hasegawa M, Kodama O, Shimamoto $\mathrm{K}$. Essential role of the small GTPase Rac in disease resistance of rice. Proc Natl Acad Sci USA. 2001;98:759-64.

Ory S, Morrison DK. Signal transduction: implications for Rasdependent ERK signaling. Curr Biol. 2004;14:R277-8.

Perfus-Barbeoch L, Jones AM, Assmann SM. Plant heterotrimeric G protein function: insights from Arabidopsis and rice mutants. Curr Opin Plant Biol. 2004;7:719-31.

Rairdan GJ, Collier SM, Sacco MA, Baldwin TT, Boettrich T, Moffett P. The coiled-coil and nucleotide binding domains of the Potato $\mathrm{Rx}$ disease resistance protein function in pathogen recognition and signaling. Plant Cell. 2008;20:739-51.

Ramezani-Rad M. The role of adaptor protein Ste50-dependent regulation of the MAPKKK Ste11 in multiple signalling pathways of yeast. Curr Genet. 2003;43:161-70.

Saijo Y, Tintor N, Lu X, Rauf P, Pajerowska-Mukhtar K, Haweker H, et al. Receptor quality control in the endoplasmic reticulum for plant innate immunity. EMBO J. 2009;28:3439-49.

Shang Y, Li X, Cui H, He P, Thilmony R, Chintamanani S, et al. RAR1, a central player in plant immunity, is targeted by Pseudomonas syringae effector AvrB. Proc Natl Acad Sci USA. 2006;103:19200-5.

Shirasu K. The HSP90-SGT1 chaperone complex for NLR immune sensors. Annu Rev Plant Biol. 2009;60:139-64.

Shiu SH, Karlowski WM, Pan R, Tzeng YH, Mayer KF, Li WH. Comparative analysis of the receptor-like kinase family in Arabidopsis and rice. Plant Cell. 2004;16:1220-34.

Suharsono U, Fujisawa Y, Kawasaki T, Iwasaki Y, Satoh H, Shimamoto K. The heterotrimeric G protein alpha subunit acts upstream of the small GTPase Rac in disease resistance of rice. Proc Natl Acad Sci USA. 2002;99:13307-12.

Takken FL, Tameling WI. To nibble at plant resistance proteins. Science. 2009;324:744-6.

Takken FL, Albrecht M, Tameling WI. Resistance proteins: molecular switches of plant defence. Curr Opin Plant Biol. 2006;9:383-90.

Tao Y, Yuan F, Leister RT, Ausubel FM, Katagiri F. Mutational analysis of the Arabidopsis nucleotide binding site-leucine-rich repeat resistance gene RPS2. Plant Cell. 2000;12:2541-54.

Thao NP, Chen L, Nakashima A, Hara S, Umemura K, Takahashi A, et al. RAR1 and HSP90 form a complex with Rac/Rop GTPase and function in innate-immune responses in rice. Plant Cell. 2007; 19:4035-45.
Thomas C, Fricke I, Scrima A, Berken A, Wittinghofer A. Structural evidence for a common intermediate in small $G$ protein-GEF reactions. Mol Cell. 2007;25:141-9.

Torres MA, Dangl JL. Functions of the respiratory burst oxidase in biotic interactions, abiotic stress and development. Curr Opin Plant Biol. 2005;8:397-403.

Triantafilou M, Miyake K, Golenbock DT, Triantafilou K. Mediators of innate immune recognition of bacteria concentrate in lipid rafts and facilitate lipopolysaccharide-induced cell activation. J Cell Sci. 2002;115:2603-11.

Trotochaud AE, Hao T, Wu G, Yang Z, Clark SE. The CLAVATA1 receptor-like kinase requires CLAVATA3 for its assembly into a signaling complex that includes KAPP and a Rho-related protein. Plant Cell. 1999;11:393-406.

Wang Y, Gao M, Li Q, Wang L, Wang J, Jeon JS, et al. OsRAR1 and OsSGT1 physically interact and function in rice basal disease resistance. Mol Plant-Microb Interact. 2008;21:294-303.

Winge P, Brembu T, Kristensen R, Bones AM. Genetic structure and evolution of RAC-GTPases in Arabidopsis thaliana. Genetics. 2000;156:1959-71.

Wong HL, Sakamoto T, Kawasaki T, Umemura K, Shimamoto K. Down-regulation of metallothionein, a reactive oxygen scavenger, by the small GTPase OsRac1 in rice. Plant Physiol. 2004;135:1447-56.

Wong HL, Pinontoan R, Hayashi K, Tabata R, Yaeno T, Hasegawa K, et al. Regulation of rice NADPH oxidase by binding of Rac GTPase to its N-terminal extension. Plant Cell. 2007;19:402234.

Yang KY, Liu Y, Zhang S. Activation of a mitogen-activated protein kinase pathway is involved in disease resistance in tobacco. Proc Natl Acad Sci USA. 2001;98:741-6.

Yuyama K, Sekino-Suzuki N, Sanai Y, Kasahara K. Translocation of activated heterotrimeric $\mathrm{G}$ protein Galpha(o) to gangliosideenriched detergent-resistant membrane rafts in developing cerebellum. J Biol Chem. 2007;282:26392-400.

Zhang Y, Dorey S, Swiderski M, Jones JD. Expression of RPS4 in tobacco induces an AvrRps4-independent HR that requires EDS1, SGT1 and HSP90. Plant J. 2004;40:213-24.

Zipfel C. Pattern-recognition receptors in plant innate immunity. Curr Opin Immunol. 2008;20:10-6.

Zipfel C, Kunze G, Chinchilla D, Caniard A, Jones JD, Boller T, et al. Perception of the bacterial PAMP EF-Tu by the receptor EFR restricts Agrobacterium-mediated transformation. Cell. 2006;125:749-60. 